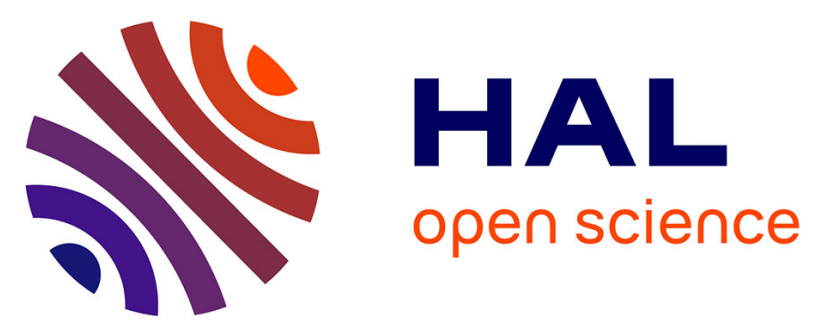

\title{
Exponential stabilization using sliding mode control for non linear systems with time-varying delays
}

\author{
Alexandre Seuret, M. Irfan, Chris Edwards, Sarah K. Spurgeon
}

\section{To cite this version:}

Alexandre Seuret, M. Irfan, Chris Edwards, Sarah K. Spurgeon. Exponential stabilization using sliding mode control for non linear systems with time-varying delays. 46th IEEE Conference on Decision and Control, 2007, Dec 2007, New Orleans, LA, United States. pp.10.1109/CDC.2007.4434453, 10.1109/CDC.2007.4434453 . hal-00386282

\section{HAL Id: hal-00386282 \\ https://hal.science/hal-00386282}

Submitted on 20 May 2009

HAL is a multi-disciplinary open access archive for the deposit and dissemination of scientific research documents, whether they are published or not. The documents may come from teaching and research institutions in France or abroad, or from public or private research centers.
L'archive ouverte pluridisciplinaire HAL, est destinée au dépôt et à la diffusion de documents scientifiques de niveau recherche, publiés ou non, émanant des établissements d'enseignement et de recherche français ou étrangers, des laboratoires publics ou privés. 


\title{
Exponential stabilization using sliding mode control for non linear systems with time-varying delays
}

\author{
A. Seuret, M. Irfan, C. Edwards and S. K. Spurgeon
}

\begin{abstract}
This paper deals with exponential stabilization of linear systems with delay using a state feedback sliding mode controller. The approach is based on Lyapunov-Krasovskii techniques and uses a descriptor representation. The exponential stability properties are proved using an appropriate change of variables associated with a polytopic representation. The system under consideration is subject to unknown, time-varying but bounded delays. The results are given in terms of LMIs. An example is given to show the efficacy of the approach.

Index Terms-Time-Varying Delay, Exponential Stability and Stabilization, LMI, Polytopic Representation, Sliding Mode Control.
\end{abstract}

\section{INTRODUCTION}

Robust control of time-delay systems is currently a topic of considerable research interest [14], [16]. Time-delays can be considered as a major cause of instability and poor performance in dynamic systems. Several results have been established for specific systems representations. Cases involving known or unknown, constant or time-varying delays, generally reduce to an LMI optimization problem. The majority of results give criteria for asymptotic stability [16] but some applications such as observer design, networked control, tele-operated systems or chained systems often require exponential convergence, in order to ensure an adequate speed of response. Recently, some authors have investigated the exponential stability of delayed systems [8],[11],[13],[15],[20]. However, these results are limited to the case of constant delay. In many situations, such as in the communication lines used in networked control, the delays cannot be reduced to the constant case. Recently [17] and [18] propose a method to take into account the variation of the delay in the stability analysis, by using a change of variable and an appropriate polytopic representation [19].

Sliding mode control is a particular type of variable structure control. Variable structure control systems (VSCS) are characterised by a suite of feedback control laws and a decision rule. The decision rule, termed the switching function, has as its input some measure of the current system behaviour and produces as an output the particular feedback controller which should be used at that instant in time. In sliding mode control, VSCS are designed to drive and then constrain the system state to lie within a neighbourhood of the switching

The Authors are with the Control and Instrumentation Research Group, Department of Engineering, University of Leicester, University Road, Leicester, LE1 7RH, UK as389, mi42, eon, ce14@le.ac.uk. This work was supported by an EPSRC Platform Grant reference EP/D029937/1 entitled 'Control of Complex Systems' and also by NESCOM. function. There are a number of advantages to this approach. Firstly, the dynamic behaviour of the system may be tailored by the particular choice of switching function. Secondly, the closed-loop response becomes totally insensitive to a particular class of uncertainty in the system.

The application of the sliding mode control methodology to the problem of systems with time-delay is not new, but the literature is limited. Generically it is a far from trivial problem involving the combination of delay phenomenon with relay actuators, and has the potential to induce oscillations around the sliding surface during the sliding mode. The problem of the development of sliding mode controllers for operation in the presence of single or multiple, constant or time-varying state delays has been solved by Gouaisbaut et al, [5]. This uses the usual regular form method of solution and the uncertainty is assumed to be matched, ie. the class of uncertainty is implicit in the range of the input channels and will be rejected by an appropriately designed sliding mode control strategy. It is important to note that in [5] full state availability is assumed. This problem has also been considered by Li and DeCarlo [12] where a class of uncertain time delay systems with multiple fixed delays in the system states are considered. The paper considers unmatched and time varying parameter uncertainties together with matched and bounded external disturbances, but again full state information is assumed to be available to the controller. Most recently, the problem has been considered by Jafarov [10], who again considers an uncertain system in the presence of fixed state-delay, and again full-state feedback is assumed. For systems where input delays are present, the problem of sliding mode control is very much open. Limited work is available such as the application specific contribution of Choi and Hedrick [1] which uses an observer based controller design framework for improving the air/fuel characteristics in engines and the work of Gouaisbaut et al. [6].

This motivates the present work, which focuses on $\alpha$ stability, exponential stability and stabilization using sliding mode control of systems subject to unknown timevarying but bounded delays. The results proposed here use a polytopic approach which allows the time-varying delay to be reduced to a convex sum of its bounds, a LyapunovKrasovskii functional and sliding mode control.

Notation : Throughout the paper, the superscript ' $T$ ' stands for matrix transposition, $\mathbb{R}^{n}$ denotes $n$-dimensional Euclidean 
space, and $\mathbb{R}^{n \times m}$ is the set of $n \times m$ real matrices. The set $I_{r}$ for all integers $r \geq 0$ contains all the integers between 1 and $r$. The notation $|$.$| and \|$.$\| refer to the Euclidean vector$ norm and the induced matrix two norm, respectively. The notation $|.| \tau_{2}$ for any function $\phi$ from $\mathscr{C}^{1}\left(\left[-\tau_{2} ; 0\right], \mathbb{R}^{n}\right)$ is $\sup _{s \in\left[-\tau_{2}, 0\right]}(|\phi(s)|)$ and $\|\phi\|_{h}=\max \left(|\phi(s)|_{\tau_{2}},|\dot{\phi}(s)|_{\tau_{2}}\right)$. The notation $P>0$ for $P \in \mathbb{R}^{n \times n}$ means that $P$ is a symmetric and positive definite matrix. Finally $I_{n}$ represents the $n \times n$ identity matrix.

\section{PROBLEM FORMULATION}

Consider the following linear system with time-varying delay and parametric uncertainties:

$$
\left\{\begin{aligned}
\dot{z_{1}}(t)= & g_{1}(z(t), t)+g_{\tau 1}(z(t-\tau(t)), t), \\
\dot{z_{2}}(t)= & g_{2}(z(t), t)+g_{\tau 2}(z(t-\tau(t)), t) \\
& +B u(t)+D f(z, t), \\
z_{1}(\theta)= & \phi_{1}(\theta), \quad \forall \theta \in\left[-\tau_{2}, 0\right], \\
z_{2}(\theta)= & \phi_{2}(\theta), \quad \forall \theta \in\left[-\tau_{2}, 0\right],
\end{aligned}\right.
$$

where $z_{1}(t) \in \mathbb{R}^{n}, z_{2}(t) \in \mathbb{R}^{m}$ represent vectors of internal variables and $u(t) \in \mathbb{R}^{m}$ is the control input vector. The function $f(z(t), t)$ represents disturbances which are assumed to satisfy:

$$
\|f(z, t)\| \leq M
$$

Assume that:

A1) $f(z, t)$ is Lipschitz continuous and satisfies the inequality

$$
\|f(z, t)\|<F_{M}(t, z),
$$

where $F_{M}$ is a continuous functional assumed to be known a priori. The functions $g_{1}, g_{\tau 1}, g_{2}$ and $g_{\tau 2}$ are written using a polytopic representation:

$$
\begin{aligned}
g_{1}(z(t), t)= & \sum_{i \in I_{r}} \lambda_{i}(t)\left\{A_{11}^{i} z_{1}(t)+A_{12}^{i} z_{2}(t)\right\}, \\
g_{\tau 1}(z(t-\tau(t), t)= & \sum_{i \in I_{r}} \lambda_{i}(t)\left\{A_{\tau 11}^{i} z_{1}(t-\tau(t))\right. \\
& \left.+A_{\tau 12}^{i} z_{2}(t-\tau(t))\right\}, \\
g_{2}(z(t), t)= & \sum_{i \in I_{r}} \lambda_{i}(t)\left\{A_{21}^{i} z_{1}(t)+A_{22}^{i} z_{2}(t)\right\}, \\
g_{\tau 2}(z(t-\tau(t), t)= & \sum_{i \in I_{r}} \lambda_{i}(t)\left\{A_{\tau 21}^{i} z_{1}(t-\tau(t))\right. \\
& \left.+A_{\tau 22}^{i} z_{2}(t-\tau(t))\right\} .
\end{aligned}
$$

The matrices $A_{1 k}^{i}, A_{\tau 1 k}^{i}, A_{2 k}^{i}, A_{\tau 2 k}^{i}$, for $i \in I_{r}$ and $k=1,2$, together with $B$ and $D$ are assumed to be known and constant with appropriate dimensions. The matrix $B$ is assumed to be nonsingular. The scalar functions $\lambda_{i}$ are not necessarily known, but they satisfy convexity properties:

$$
\begin{array}{ll}
\sum_{i \in I_{r}} \lambda_{i}(t)=1, & \forall t \geq 0, \\
\lambda_{i}(t) \geq 0, & \forall t \geq 0 \text { and } \forall i \in I_{r} .
\end{array}
$$

The function $\tau(t)$ represents a time-varying delay satisfying the following inequalities:

$$
0 \leq \tau(t) \leq \tau_{2}
$$

A state feedback sliding mode control law is sought which exponentially stabilizes system (1), so that there exist positive numbers $\alpha$ and $\beta>1$ such that the solution $x\left(t ; t_{0}, \phi\right)$ of the system satisfies, for any initial function $\phi$ :

$$
\left|x\left(t ; t_{0}, x_{0}\right)\right|<\beta\|\phi(s)\|_{\tau_{2}} e^{-\alpha\left(t-t_{0}\right)} .
$$

\section{EXPONENTIAL STABILITY CONDITIONS FOR TIME DELAY SYSTEMS}

In this section, consider the following time-delay system:

$$
\dot{x}(t)=\sum_{i \in I_{r}} \lambda_{i}(t)\left\{A_{0}^{i} x(t)+A_{1}^{i} x(t-\tau(t))\right\} .
$$

To develop exponential stability conditions, a change of variables will be employed as previously adopted in [17], [20]. With the new variable $x_{\alpha}=x(t) e^{\alpha t}$, the differential equations of the initial system (7) can be written as:

$$
\begin{gathered}
\dot{x}_{\alpha}(t)=\sum_{i \in I_{r}} \lambda_{i}(t)\left\{\left(A_{0}^{i}+\alpha I\right) x_{\alpha}(t)+e^{\alpha \tau(t)} A_{1}^{i} x_{\alpha}(t-\tau(t))\right\} . \\
x_{\alpha}(s)=e^{\alpha s} \phi(s), \quad \forall s \in\left[-\tau_{2}, 0\right]
\end{gathered}
$$

Asymptotic stability of system (8) for some $\alpha>0$ implies $\alpha$-stability of system (7).

The main difficulty which appears here stems from the fact that the system (8) is time-varying because of the gain $e^{\alpha \tau(t)}$ which appears with the delayed term $A_{1}$. In the case of a constant delay, the change of variable only adds gain modifications to the asymptotic stability conditions and then it becomes very easy to ensure exponential stability. However in the case of time-varying delays, the conditions are no longer applicable. As the delay is assumed to be bounded as in (5), the exponential gain $e^{\alpha \tau(t)}$ can be rewritten using a polytopic representation as in [17]. This approach uses the fact that the function $e^{\alpha \tau(t)}$ is bounded. Knowing the bounds of the delay $\tau(t)$ as given in (5), the term $e^{\alpha \tau(t)}$ can be written as a convex sum of its bound $\beta_{1}=e^{\alpha 0}=1$ and $\beta_{2}=e^{\alpha \tau_{2}}$ :

$$
\begin{aligned}
& e^{\alpha \tau(t)}=\mu_{1}(t) \beta_{1}+\mu_{2}(t) \beta_{2}, \\
& \mu_{1}(t), \mu_{2}(t) \geq 0 \text { and } \mu_{1}(t)+\mu_{2}(t)=1,
\end{aligned}
$$

where the scalar functions $\mu_{1}(t)=\left(\beta_{2}-e^{\alpha \tau(t)}\right) /\left(\beta_{2}-\beta_{1}\right)$ and $\mu_{2}(t)=\left(e^{\alpha \tau(t)}-\beta_{1}\right) /\left(\beta_{2}-\beta_{1}\right)$ only depend on the unknown delay value $\tau(t)$. Consequently these functions are thus also unknown but they satisfy the convexity conditions (9). By using the first equation of (9), the system (8) can be expressed in the following polytopic form:

$$
\begin{aligned}
\dot{x}_{\alpha}(t)= & \sum_{i \in I_{r}} \lambda_{i}(t)\left\{\left(A_{0}^{i}+\alpha I_{n}\right) x_{\alpha}(t)\right. \\
& \left.+\sum_{j=1}^{2} \mu_{j}(t) \beta_{j} A_{1}^{i} x_{\alpha}(t-\tau(t))\right\},
\end{aligned}
$$

or

$$
\begin{aligned}
\dot{x}_{\alpha}(t)=\quad & \sum_{i \in I_{r}, j=1}^{2} \bar{\lambda}_{j}^{i}(t)\left\{\left(A_{0}^{i}+\alpha I_{n}\right) x_{\alpha}(t)\right. \\
& \left.+\beta_{j} A_{1}^{i} x_{\alpha}(t-\tau(t))\right\},
\end{aligned}
$$

where $\bar{\lambda}_{j}^{i}(t)=\lambda_{i}(t) \mu_{j}(t)$, for all $i \in I_{r}$ and $j=1,2$. Applying the Newton-Leibniz formula:

$$
\begin{aligned}
\dot{x}_{\alpha}(t)= & \sum_{i \in I_{r}, j=1}^{2} \bar{\lambda}_{j}^{i}(t)\left\{\left(A_{0}^{i}+\alpha I_{n}+\beta_{j} A_{1}^{i}\right) x_{\alpha}(t)\right. \\
& \left.-\beta_{j} A_{1}^{i} \int_{t-\tau(t)}^{t} \dot{x}_{\alpha}(s) d s\right\} .
\end{aligned}
$$

To analyse the asymptotic stability properties of this system, equation (11) can be rewritten using the descriptor representation introduced in [2], [4]:

$$
\begin{aligned}
E \dot{\bar{x}}_{\alpha}(t)= & \sum_{i \in I_{r}, j=1}^{2} \bar{\lambda}_{j}^{i}(t)\left\{\left[\begin{array}{cc}
0 & I_{n} \\
\Lambda_{j}^{i} & -I_{n}
\end{array}\right] \bar{x}_{\alpha}(t)\right. \\
& \left.-\left[\begin{array}{c}
0 \\
\beta_{j} A_{1}^{i}
\end{array}\right] \int_{t-\tau(t)}^{t} \dot{x}_{\alpha}(s) d s\right\}
\end{aligned}
$$


with $\bar{x}_{\alpha}(t)=\operatorname{col}\left\{x_{\alpha}(t), \dot{x}_{\alpha}(t)\right\}, E=\operatorname{diag}\left\{I_{n}, 0\right\}$ and $\Lambda_{j}^{i}=$ $\alpha I_{n}+A_{0}^{i}+\beta_{j} A_{1}^{i}$. Then the following result holds:

Theorem 1: The system (7) is $\alpha$-stable for all time-varying delays $0 \leq \tau(t) \leq \tau_{2}$, if there exist $n \times n$ matrices $P_{2}, P_{3}, Z_{1}^{i j}$, $Z_{2}^{i j}, Z_{3}^{i j}$, for $i=1,2$, and two symmetric definite matrices $P_{1}>0$ and $R>0$ such that the following LMI conditions are satisfied for $i \in I_{r}$ and $j=1,2$ :

$$
\Psi_{j}^{i}<0
$$

and

$$
\left[\begin{array}{ll}
R & {\left[\begin{array}{cc}
0 & \beta_{j} A_{1}^{i T}
\end{array}\right] P} \\
* & Z^{i j}
\end{array}\right] \geq 0
$$

where

$$
P=\left[\begin{array}{cc}
P_{1} & 0 \\
P_{2} & P_{3}
\end{array}\right], \quad Z^{i j}=\left[\begin{array}{cc}
Z_{1}^{i j} & Z_{2}^{i j} \\
Z_{2}^{i j T} & Z_{3}^{i j}
\end{array}\right]
$$

and

$$
\begin{aligned}
\Psi_{j}^{i}= & P^{T}\left[\begin{array}{cc}
0 & I_{n} \\
\Lambda_{j}^{i} & -I_{n}
\end{array}\right]+\left[\begin{array}{cc}
0 & I_{n} \\
\Lambda_{j}^{i} & -I_{n}
\end{array}\right]^{T} P \\
& +\tau_{2} Z^{i j}+\left[\begin{array}{cc}
0 & 0 \\
0 & \tau_{2} R
\end{array}\right],
\end{aligned}
$$
tional:

Proof: Define the following Lyapunov-Krasovskii func-

$$
V\left(\bar{x}_{\alpha}(t)\right)=\bar{x}_{\alpha}^{T}(t) E P \bar{x}_{\alpha}(t)+\int_{-\tau_{2}}^{0} \int_{t+\theta}^{t} \dot{x}_{\alpha}^{T}(s) R \dot{x}_{\alpha}(s) d s d \theta
$$

This functional is positive definite as $\bar{x}_{\alpha}^{T}(t) E P \bar{x}_{\alpha}(t)=$ $x_{\alpha}^{T}(t) P_{1} x_{\alpha}(t)$. Then noting that $E P=P^{T} E$, the computation of the time-derivative yields:

$$
\begin{aligned}
\dot{V}(t)= & \sum_{i \in I_{r}, j=1}^{2} \bar{\lambda}_{j}^{i}(t)\left\{\bar{x}_{\alpha}^{T}(t) \Psi_{0 j}^{i} \bar{x}_{\alpha}(t)+\eta_{0 j}^{i}(t)\right\} \\
& +\tau_{2} \dot{x}_{\alpha}^{T}(t) R \dot{x}_{\alpha}(t)-\int_{t-\tau_{2}}^{t} \dot{x}_{\alpha}^{T}(s) R \dot{x}_{\alpha}(s) d s
\end{aligned}
$$

where

$$
\begin{aligned}
& \Psi_{0 j}^{i}=P^{T}\left[\begin{array}{cc}
0 & I_{n} \\
\Lambda_{j}^{i} & -I_{n}
\end{array}\right]+\left[\begin{array}{cc}
0 & I_{n} \\
\Lambda_{j}^{i} & -I_{n}
\end{array}\right]^{T} P, \\
& \eta_{0 j}^{i}(t)=-2 \int_{t-\tau(t)}^{t} \bar{x}_{\alpha}^{T}(t) P^{T}\left[\begin{array}{c}
0 \\
\beta_{j} A_{1}^{i}
\end{array}\right] \dot{x}_{\alpha}(s) d s .
\end{aligned}
$$

The function $\eta_{0 j}^{i}$ has to be bounded to guarantee the negativity of $\dot{V}$. Define the following linear matrix inequality:

$$
\left[\begin{array}{cc}
R & N \\
N^{T} & Z
\end{array}\right] \geq 0
$$

which ensures, for all vectors $a$ and $b$ and for all matrices $N$, $R$ and $Z$ of appropriate dimensions, the following inequality holds :

$$
\begin{aligned}
& {\left[\begin{array}{l}
a \\
b
\end{array}\right]^{T}\left[\begin{array}{cc}
R & N \\
N^{T} & Z
\end{array}\right]\left[\begin{array}{l}
a \\
b
\end{array}\right] \geq 0} \\
& \Leftrightarrow \pm 2 a^{T} N^{T} b<a^{T} R a+b^{T} Z b
\end{aligned}
$$

As $\eta_{0 j}^{i}$ is already written in a polytopic form, it is also necessary to find a bound for each polytope, for each subsystem. Let $N=\left[\begin{array}{cc}0 & \beta_{j} A_{1}^{i T}\end{array}\right] P$ and suppose $R$ and $Z^{i j}$ satify:

$$
\left[\begin{array}{ccc}
R & {\left[\begin{array}{cc}
0 & \beta_{j} A_{1}^{i T}
\end{array}\right] P} \\
* & & Z^{i j}
\end{array}\right] \geq 0, \quad i \in I_{r} \text { and } j=1,2
$$

which, together with the convexity properties in (9), $a=$ $\dot{x}_{\alpha}(s)$ and $b=\bar{x}_{\alpha}(t)$, yields:

$$
\eta_{0 j}^{i}(t) \leq \int_{t-\tau(t)}^{t}\left\{\bar{x}_{\alpha}^{T}(t) Z^{i j} \bar{x}_{\alpha}(t)+\dot{x}_{\alpha}^{T}(s) R \dot{x}_{\alpha}(s)\right\} d s,
$$

Integrating with respect to the variable " $s$ " from $t-\tau_{2}$ to $t$ and applying (5) yields:

$$
\begin{array}{r}
\sum_{i \in I_{r}, j=1}^{2} \bar{\lambda}_{j}^{i}(t) \eta_{0 j}^{i}(t) \leq \int_{t-\tau_{2}}^{t} \dot{x}_{\alpha}^{T}(s) R \dot{x}_{\alpha}(s) d s \\
+\tau_{2} \bar{x}_{\alpha}^{T}(t)\left(\sum_{i \in I_{r}, j=1}^{2} \bar{\lambda}_{j}^{i}(t) Z^{i j}\right) \bar{x}_{\alpha}(t)
\end{array}
$$

with $R, Z^{i 1}$ and $Z^{i 2}$ satisfying (12). Then it follows:

$$
\dot{V}(t) \leq \bar{x}_{\alpha}^{T}(t) \Psi(t) \bar{x}_{\alpha}(t),
$$

where

$$
\begin{aligned}
\Psi(t)= & \sum_{i \in I_{r}, j=1}^{2} \bar{\lambda}_{j}^{i}(t)\left\{P^{T}\left[\begin{array}{cc}
0 & I_{n} \\
\Lambda_{j}^{i} & -I_{n}
\end{array}\right]\right. \\
& \left.+\left[\begin{array}{cc}
0 & I_{n} \\
\Lambda_{j}^{i} & -I_{n}
\end{array}\right]^{T} P+\tau_{2} Z^{i j}\right\}+\left[\begin{array}{cc}
0 & 0 \\
0 & \tau_{2} R
\end{array}\right]
\end{aligned}
$$

By rewriting as a polytopic sum, $\dot{V}<0$ if:

$$
\sum_{i \in I_{r}, j=1}^{2} \bar{\lambda}_{j}^{i}(t) \Psi_{j}^{i}<0
$$

If each individual matrix $\Psi_{j}^{i}$ is negative definite, $\Psi(t)$ will also be negative definite and the transformed system (8) is asymptotically stable. Consequently, the original system (7) is exponentially stable with a guaranteed decay rate $\alpha$.

\section{SLIDING MODE CONTROLlER}

In this section, time delay systems that can be rewritten in the form (1) are considered. The time-varying functions $\lambda_{i}$ are not necessarily known, but are assumed to be convex. Consider the following switching function :

$$
s(z)=z_{2}-K z_{1} .
$$

where $K \in \mathbb{R}^{m \times n}$ is a gain to be defined.

A. Sliding mode control design for a system with state delay Denote

$$
\begin{aligned}
& \Theta\left(t, z_{t}\right)=g_{2}\left(z_{t}, t\right)-K g_{1}\left(z_{t}, t\right) \\
& \left.D_{M}\left(z_{t}\right)=g_{d 2}(t-v, z(t-v))-K g_{d 1}(t-v, z(t-v))\right)
\end{aligned}
$$

The function $\Theta\left(t, z_{t}\right)$ can be bounded by using the convex properties of the scalar functions $\lambda_{i}$. It follows that:

$$
\left\|\Theta\left(t, z_{t}\right)\right\| \leq D_{1}(t)=\sum_{k=1}^{2} \max _{i \in I_{r}}\left\{\left\|A_{2 k}^{i}-K A_{1 k}^{i}\right\|\right\}\left\|z_{k}(t)\right\| .
$$

Using the same technique:

$$
\begin{aligned}
\left\|D_{M}\left(z_{t}\right)\right\| \leq D_{2}(t)= & \sum_{k=1}^{2} \max _{i \in I_{r}}\left\{\left\|A_{\tau 2 k}^{i}-K A_{\tau 1 k}^{i}\right\|\right\} \\
& \times \sup _{0<s<\tau_{2}}\left\|z_{k}(t-s)\right\|
\end{aligned}
$$

Theorem 2: Consider system (1). If for all time-varying delay $\tau(t)$ satisfying (5), there exist $n \times n$ matrices $Q_{1}>0$, $S>0 Q_{2}, Q_{3}, W_{1}^{i j}, W_{2}^{i j}, W_{3}^{i j}$ and a $m \times n$ matrix $Y$ such 
that the following LMI conditions are satisfied for $i \in I_{r}$ and $j=1,2$ :

$$
\begin{aligned}
& {\left[\begin{array}{ccc}
Q_{2}+Q_{2}^{T}+\tau_{2} W_{1}^{i j} & \Phi_{12}^{i j} & \tau_{2} Q_{2}^{T} \\
* & -Q_{3}-Q_{3}^{T}+\tau_{2} W_{3}^{i j} & \tau_{2} Q_{3}^{T} \\
* & * & -\tau_{2} S
\end{array}\right]<0,} \\
& {\left[\begin{array}{ccc}
2 Q_{1}-S & 0 & \beta_{j}\left(Q_{1} A_{\tau 11}^{i T}+Y^{T} A_{\tau 12}^{i T}\right) \\
* & W_{1}^{i j} & W_{2}^{i j} \\
* & * & W_{3}^{i j}
\end{array}\right] \geq 0,}
\end{aligned}
$$

where $\Phi_{12}^{i j}=Q_{1}\left(A_{11}^{i}+\alpha I_{n}+\beta_{j} A_{\tau 11}^{i}\right)^{T}+Y^{T}\left(A_{12}^{i}+\right.$ $\left.\beta_{j} A_{\tau 12}^{i}\right)^{T}-Q_{2}^{T}+Q_{3}+\tau_{2} W_{2}^{i j}$, the sliding mode gain is given by $K=Y Q_{1}^{-1}$ and, for any Hurwitz Matrix $G_{l}$, the control law :

$$
\begin{aligned}
u(t)= & -B^{-1}\left(-G_{l} s(t)+\left[D_{1}(t)+D_{2}(t)\right.\right. \\
& \left.\left.+\|D\| F_{M}(z, t)+\delta\right] \frac{s(t)}{\|s(t)\|}\right)
\end{aligned}
$$

ensures the systems trajectories reach the sliding manifold $s(t)=0$ in finite time. Furthermore the solutions converge exponentially.

Proof: The proof is divided into two parts. The first part deals with the proof of the existence of an ideal sliding motion on the surface $s(z)=0$ and the second with the $\alpha$-stability of the reduced system.

To demonstrate the attractivity of the set " $s(z(t))=0$ ", the following Lyapunov-Krasovskii functional is used:

$$
V(t)=s^{T}(z(t)) s(z(t))=\|s(z(t))\|^{2} .
$$

Differentiating (23), along the trajectories of the closed-loop system gives

$$
\begin{aligned}
\dot{V}(t)= & 2 s^{T}(t)\left[g_{2}(z(t), t)+K g_{1}\left(z_{t}, t\right)\right. \\
& \left.+g_{d 2}(z(t-h(t)), t)+K g_{d 1}(z(t-h(t)), t)\right) \\
& +D f(z, t)+B u(t)]
\end{aligned}
$$

Using the control law (22), it follows that:

$$
\dot{V}(t) \leq-2 \delta\|s(z(t))\|=-2 \delta V(t)^{\frac{1}{2}}
$$

The last inequality is known to prove the finite time convergence of the system (1) to the surface $s=0$.

Substituting for $A_{0}^{i}$ and $A_{1}^{i}$ using $A_{11}^{i}, A_{\tau 11}^{i}, A_{\tau 12}^{i}$ and $A_{\tau 12}^{i}$, it follows that:

$$
\dot{z}_{1}(t)=\left(A_{11}^{i}+A_{12}^{i} K\right) z_{1}(t)+\left(A_{\tau 11}^{i}+A_{\tau 12}^{i} K\right) z_{1}(t-\tau(t)) .
$$

From Theorem 1, the $\alpha$-stability of (24) is proved if the following inequalities are satisfied for $i=1,2$ :

$$
\Psi_{j}^{i}<0, \quad\left[\begin{array}{cc}
R & {\left[\begin{array}{cc}
0 & \beta_{j}\left(A_{\tau 11}^{i}+A_{\tau 12}^{i} K\right)^{T}
\end{array}\right] P} \\
* & Z^{i j}
\end{array}\right] \geq 0
$$

with

$$
\begin{aligned}
P= & {\left[\begin{array}{cc}
P_{1} & 0 \\
P_{2} & P_{3}
\end{array}\right], \quad Z^{i j}=\left[\begin{array}{cc}
Z_{1}^{i j} & Z_{2}^{i j} \\
Z_{2}^{i j T} & Z_{3}^{i j}
\end{array}\right], } \\
\Psi_{j}^{i}= & P^{T}\left[\begin{array}{cc}
0 & I_{n} \\
\Lambda_{j}^{i} & -I_{n}
\end{array}\right]+\left[\begin{array}{cc}
0 & I_{n} \\
\Lambda_{j}^{i} & -I_{n}
\end{array}\right]^{T} P \\
& +\tau_{2} Z^{i j}+\left[\begin{array}{cc}
0 & 0 \\
0 & \tau_{2} R
\end{array}\right], \\
\Lambda_{j}^{i}= & \alpha I_{n}+A_{11}^{i}+A_{\tau 12}^{i} K+\beta_{j}\left(A_{\tau 11}^{i}+A_{\tau 12}^{i} K\right) .
\end{aligned}
$$

Note that these conditions are not LMIs because of product terms involving $K$ and $P$. The following exposition deals with the transformation of these inequalities into LMIs. First, following the proof of [3], the previous conditions are developed and modified by using the Schur complement where $\operatorname{diag}\left(0, \tau_{2} R\right)$ is written as:

$$
\left[\begin{array}{cc}
0 & 0 \\
0 & \tau_{2} R
\end{array}\right]=\tau_{2}\left[\begin{array}{c}
0 \\
I_{n}
\end{array}\right]\left(\tau_{2} R\right) \tau_{2}\left[\begin{array}{c}
0 \\
I_{n}
\end{array}\right]^{T} .
$$

Note that $P_{1}$ and $\left(P_{3}^{T}+P_{3}\right)$ must be positive definite to guarantee the negativity of $\Psi_{j}^{i}$ in (12). Consequently the matrix $P$ is nonsingular. Define the matrix $Q$ as follows :

$$
Q=\left[\begin{array}{cc}
Q_{1} & 0 \\
Q_{2} & Q_{3}
\end{array}\right]=P^{-1} .
$$

By applying the Schur complement to (26) and by multiplying the previous LMIs by $\Delta_{1}=\operatorname{diag}\left\{Q, I_{2 n \times 2 n}\right\}$ and $\Delta_{1}^{T}$ respectively on the right and on the left, the definitions

$$
W^{i j}=Q^{T} Z^{i j} Q=\left[\begin{array}{cc}
W_{1}^{i j} & W_{2}^{i j} \\
* & W_{3}^{i j}
\end{array}\right]
$$

and $S=R^{-1}$ lead to:

$$
\left[\begin{array}{ccc}
Q_{2}+Q_{2}^{T}+W_{1}^{i j} & \Theta_{21} & \tau_{2} Q_{2}^{T} \\
* & -Q_{3}-Q_{3}^{T}+W_{3}^{i j} & \tau_{2} Q_{3}^{T} \\
* & * & -\tau_{2} S
\end{array}\right]<0,
$$

where

$$
\begin{aligned}
\Theta_{21}= & Q_{1}\left(A_{11}^{i}+\alpha I_{n}+\beta_{j} A_{\tau 11}^{i}\right)^{T}-Q_{2}^{T}+Q_{3} \\
& +Q_{1} K^{T}\left(A_{12}^{i}+\beta_{j} A_{\tau 12}^{i}\right)^{T}+W_{2}^{i j} .
\end{aligned}
$$

The second LMI condition of Theorem 1 must be expressed in terms of the same variables defined above. Multiply (13) by $\operatorname{diag}\left\{Q_{1}, Q^{T}\right\}$ on the left and by its transpose on the right leads to the following nonlinear inequality:

$$
\left[\begin{array}{ccc}
Q_{1}^{T} S^{-1} Q_{1} & 0 & \beta_{j} Q_{1}\left(A_{\tau 11}^{i}+A_{\tau 12}^{i} K\right)^{T} \\
* & W_{1}^{i j} & W_{2}^{i j} \\
* & * & W_{3}^{i j}
\end{array}\right] \geq 0 .
$$

The term $Q_{1}^{T} S^{-1} Q_{1}$ cannot be computed directly. Adding an additional constraint such that $Q_{1}=\varepsilon S$ can solve the problem directly, but this also leads to conservative results. Alternatively, the following inequality, introduced in [7] $\left(Q_{1}-S\right)^{T} S^{-1}\left(Q_{1}-S\right) \geq 0$ can be used which ensures $-Q_{1} S^{-1} Q_{1} \leq-2 Q_{1}+S$.

Denoting $D_{j}^{i}=\beta_{j}\left(A_{\tau 11}^{i}+A_{\tau 12}^{i} K\right) Q_{1}$, then the Schur complement enables (28) to be expressed as:

$$
-Q_{1} S Q_{1}+\left[\begin{array}{c}
0 \\
D_{j}^{i}
\end{array}\right]^{T}\left(W^{i j}\right)^{-1}\left[\begin{array}{c}
0 \\
D_{j}^{i}
\end{array}\right]<0 .
$$

If the condition:

$$
-2 Q_{1}+S+\left[\begin{array}{c}
0 \\
D_{j}^{i}
\end{array}\right]^{T}\left(W^{i j}\right)^{-1}\left[\begin{array}{c}
0 \\
D_{j}^{i}
\end{array}\right]<0,
$$

is satisfied, then (29) will also be satisfied. By applying the Schur complement, (21) is obtained. Defining $Y=K Q_{1}$ finally ensures that (20) and (21) guarantee exponential stability of the system (24) on the sliding manifold. 
Remark 1: Note that in the case where the scalar functions $\lambda_{i}$ are known, the conservatism of the result can be reduced by defining a sliding surface for each subsystem $i \in I_{r}$ using $Y^{i}$ (and furthermore $K^{i}$ ) in Theorem 2. Then it is also possible to reduce the gain of the discontinuous term by modifying the control law $u(t)$ defined in (22) to:

$$
\begin{aligned}
u(t)= & -B^{-1}\left[-G_{l} s(t)+\sum_{k=1}^{2} \lambda_{i}(t)\left\{A_{2 k}^{i}-K^{i} A_{1 k}^{i}\right\} z_{k}(t)\right. \\
& \left.+\left(D_{2}(t)+\|D\| F_{M}(z, t)+\delta\right) \frac{s(t)}{\|s(t)\|}\right],
\end{aligned}
$$

\section{B. Gain optimization}

As the controller depends on the variable $s$, it is a interesting to minimize the value of the gain $K$ defined in Theorem 2 . Consider minimization of $K K^{T}$. As $K$ depends on the LMI variables $Q_{1}$ and $Y$ the minimization of such a function is not straightforward. However introducing an additional LMI variable $c$ such that $K K^{T}<c I_{m}$ or equivalently $-c I_{m}+$ $Y Q_{1}^{-1} I_{n} Q_{1}^{-1} Y^{T}<0$ will help to solve this problem. Consider the following result. For any matrices $A, P_{0}>0$ and $P_{1}>0$, the following equivalence holds [9]:

$$
\begin{gathered}
-P_{0}+A^{T} P_{1} A<0 \Leftrightarrow \\
\exists X \in \mathbb{R}^{n \times n},\left[\begin{array}{cc}
-P_{0} & A^{T} X^{T} \\
X A & -X-X^{T}+P_{1}
\end{array}\right]<0 .
\end{gathered}
$$

Applying this result to the optimization problem and replacing $P_{0}, P_{1}$ and $A$ by $c I_{m}, I_{n}$ and $Q_{1}^{-1} Y^{T}$ and choosing $X=Q_{1}$ leads to the LMI:

$\min c$ subject to (20) and (21) and:

$$
\left[\begin{array}{cc}
-c I_{m} & Y \\
* & -2 Q_{1}+I_{n}
\end{array}\right]<0 .
$$

\section{Example}

Consider system (1) with:

$$
\begin{aligned}
& A_{11}^{1}=\left[\begin{array}{cc}
-2.07 & -1 \\
-0.6 & 0.1
\end{array}\right], \quad A_{12}^{1}=\left[\begin{array}{c}
-1 \\
0
\end{array}\right], \\
& A_{11}^{2}=\left[\begin{array}{cc}
-2 & -1 \\
-0.4 & 0.1
\end{array}\right], \quad A_{12}^{2}=\left[\begin{array}{c}
-1.7 \\
0
\end{array}\right] \text {, } \\
& A_{\tau 11}^{1}=\left[\begin{array}{cc}
-0.2 & -.9 \\
-0.9 & -0.55
\end{array}\right], \quad A_{\tau 12}^{1}=\left[\begin{array}{c}
0 \\
0.1
\end{array}\right] \text {, } \\
& A_{\tau 11}^{2}=\left[\begin{array}{cc}
-0.2 & -0.9 \\
-0.9 & -0.55
\end{array}\right], \quad A_{\tau 12}^{2}=\left[\begin{array}{c}
0 \\
0.1
\end{array}\right] \text {, } \\
& A_{21}^{1}=A_{21}^{2}=\left[\begin{array}{ll}
-1.9 & 1
\end{array}\right], \quad A_{22}^{1}=A_{22}^{2}=-2.1 \text {, } \\
& A_{\tau 21}^{1}=A_{\tau 21}^{2}=\left[\begin{array}{ll}
-0.1 & 0.1
\end{array}\right], \quad A_{\tau 22}^{1}=A_{\tau 22}^{2}=0.9
\end{aligned}
$$

Figure 1. shows the relation between $\tau_{2}$ and the maximum exponential decay rate $\alpha$ using Theorem 2 .

For $\tau_{2}=0.2$ and $\alpha=1.5$, Theorem 2 and the optimization problem (30) ensures the sliding mode controller (22) exponentially stabilizes the system and the resulting gain is $K=\left[\begin{array}{ll}2.9199 & -5.8823\end{array}\right]$ (instead of $K=\left[\begin{array}{l}4.3196-8.3191\end{array}\right]$ without optimization).

For $\tau_{2}=0.2$ and $\alpha=0.5$, Theorem 2 and the optimization problem (30) ensures the sliding mode controller (22) exponentially stabilizes the system and the resulting gain is $K=[0.3912-2.4650]$ (instead of $K=[1.0674-4.0708]$ without optimization). Figures 2. and 3. show the simulations results for $\tau_{2}=0.2$ and for $\alpha=0.5$ and 1.5:

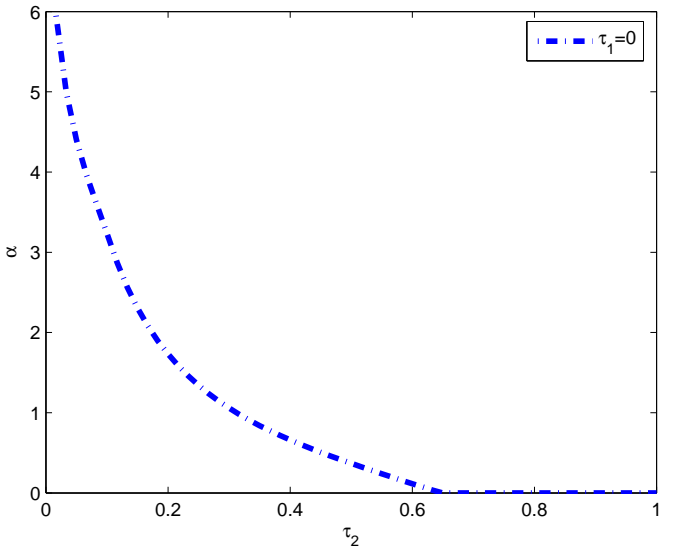

Fig. 1. Relation between $\tau_{2}$ and $\alpha_{\max }$
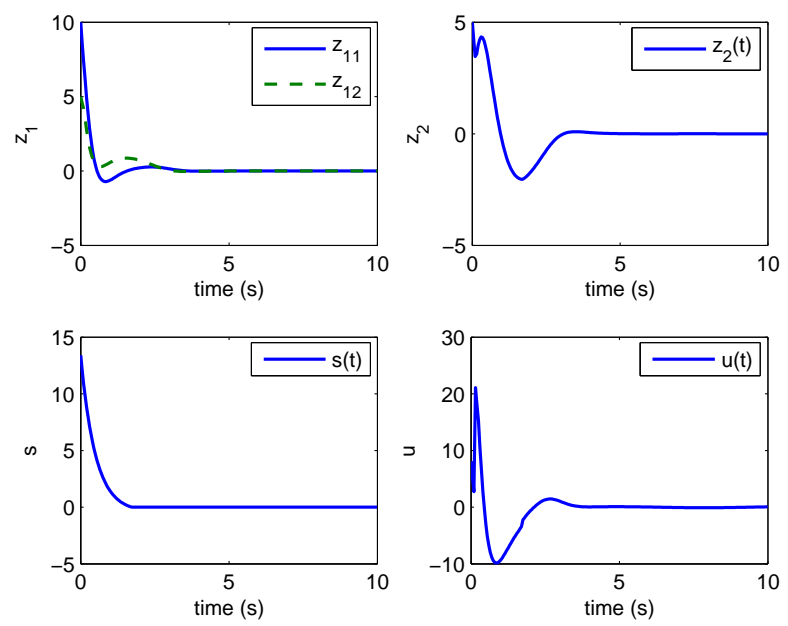

Fig. 2. Simulation for $\alpha=0.5$ and $\tau_{2}=0.2$
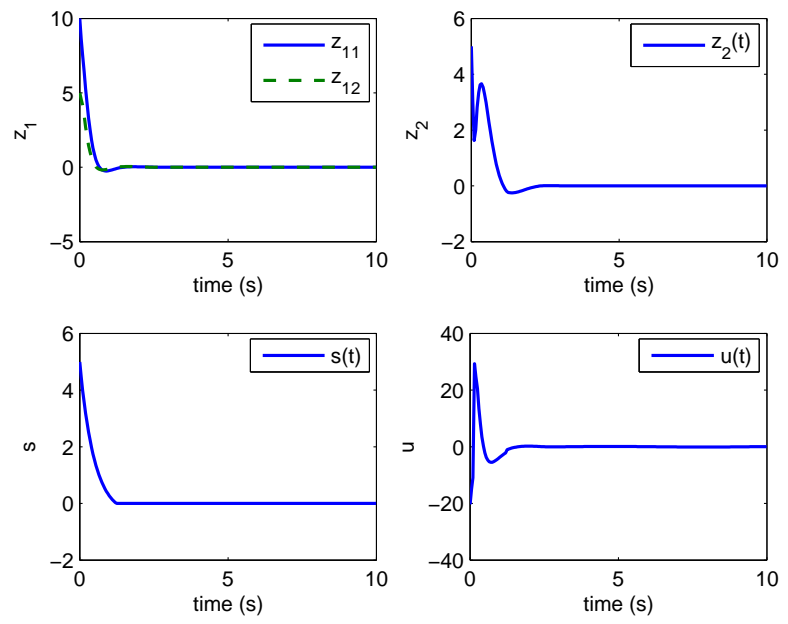

Fig. 3. Simulation for $\alpha=1.5$ and $\tau_{2}=0.2$

The main difference between the two simulations is in the 
cost of the control function. Increasing the exponential decay rate $\alpha$ produces a faster response as expected but it increases the energy required by the controller.

\section{CONCLUSION}

This paper has considered the exponential stabilization of linear, uncertain systems in the presence of unknown timevarying delays. Using a classical switching function, the existence of an ideal sliding mode and the $\alpha$-stability of the reduced order sliding mode dynamics is proved. The proofs are based on Lyapunov-Krasovskii methods and particular parameterizations. The approach is constructive and the method has been demonstrated on a numerical example.

\section{REFERENCES}

[1] S.B. Choi and J.K.Hedrick, An observer based controller design method for improving air/fuel characterisation of spark ignition engines, IEEE Trans. on Control Systems Technology 6 (1998), 325-334.

[2] E. Fridman, New Lyapunov-Krasovskii functionals for stability of linear retarded and neutral type systems, System and Control Letters 43 (2001), 309-319.

[3] E. Fridman, F. Gouaisbaut, M. Dambrine, and J.-P. Richard, Sliding mode control of systems with time-varying delays via descriptor approach, proceedings of ECC'03 (Cambridge, England), 2003.

[4] E. Fridman and U. Shaked, A descriptor system approach to $H^{\infty}$ control of linear time-delay systems, IEEE Trans. on Automatic Control 47 (2002), no. 2, 253-270.

[5] F. Gouaisbaut, M. Dambrine, and J.-P. Richard, Robust control of dealy systems: a sliding mode control design via LMI, Systems and Control Letters 46 (2002), no. 4, 219-230.

[6] F. Gouaisbaut, W. Perruquetti, and J.P.Richard, A sliding mode control for linear systems with input and state delays, Proceedings of the 38th IEEE CDC (Phoenix, Arizona), 1999, pp. 4234-4239.

[7] T.-M. Guerra, A. Kruszewski, L. Vermeiren, and H. Tirmant, Conditions of output stabilization for nonlinear models in the TakagiSugeno's form, Fuzzy Sets \& Systems 157 (2006), no. 9, 1248-1259.
[8] C. Hou and J. Qian, On an estimate of the decay rate for applications of razumikhin-type theorems, IEEE Transactions on Automatic Control 43 (1998), no. 7, 958-960.

[9] L.-S. Hu, J. Huang, and H.-H. Cao, Robust digital model predicitve control for linear uncertain systems with saturations, IEEE Trans. on Automatic Control 49 (2004), no. 5, 792-796.

[10] E. M. Jafarov, Robust sliding mode controllers design techniques for stabilization of multivariable time-delay systems with parameter perturbations and external disturbances, Int. J. Systems Science 36 (2005), no. 7, 433-444.

[11] B. Lehman and K. Shjaee, delay independant stability coditions and decay estimates for time-varying functionnal differential equations, IEEE Transactions on Automatic Control 39 (1994), no. 8, 1973-1676.

[12] X. Li and R.A. DeCarlo, Robust sliding mode control of uncertain time delay systems, Int. J. Control 76 (2003), no. 3, 1296-1305.

[13] P.-L. Liu, Robust exponential stabilization for uncertain systems with state and control delay, International Journal of Systems Science 34 (October 2003), no. 12-13, 675-682.

[14] S.-I. Niculescu, Delay effects on stability. a robust control approach, Springer-Verlag, 2001.

[15] S.-I. Niculescu, C.-E. de Souza, L. Dugard, and J.-M. Dion, Robust exponential stability of uncertain systems with time-varying delays, Proceedings of the 33rd IEEE CDC, 1994, p. 431.

[16] J.-P. Richard, Time delay systems: an overview of some recent advances and open problems, Automatica 39 (2003), 1667-1694.

[17] A. Seuret, M. Dambrine, and J.-P. Richard, Robust exponential stabilization for systems with time-varying delays, 5th Workshop on Time Delay Systems, September 2004.

[18] A. Seuret, E. Fridman, and J.-P. Richard, Sampled-data exponential stabilization of neutral systems with input and state delays, IEEE MED 2005, $13^{\text {th }}$ Mediterranean Conference on Control and Automation, June 2005.

[19] Y. Xia and Y. Jia, Robust control of state delayed systems with politopic uncertainties via parameter-dependent lyapunov functionals, Systems and Control Letters 50 (2003), 183-193.

[20] S. Xu, J. Lam, and M. Zhong, New exponential estimates for timedelay systems, IEEE Transactions on Automatic Control 51 (2006), no. $9,1501-1505$. 